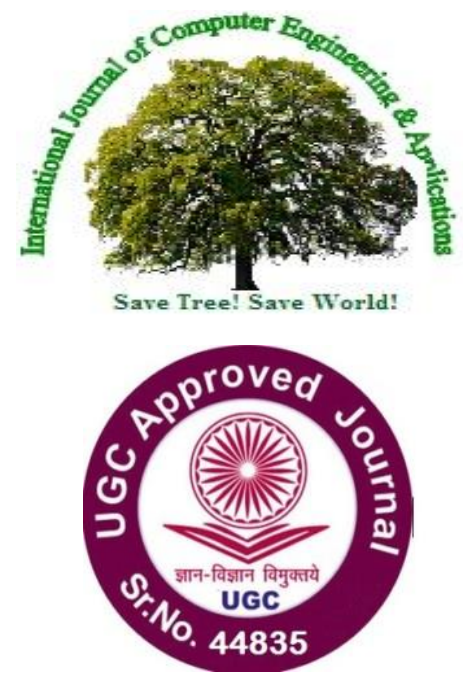

\title{
A STUDY OF 3D INTERNET AND ITS ROLE IN TECHNOLOGY WORLD
}

\author{
Tejas Sawant ${ }^{\mathbf{1}}$, Sarang Gulavni ${ }^{2}$, Dr. B.V Patil ${ }^{3}$, Prof. Nripesh Nrip ${ }^{\mathbf{4}}$ \\ ${ }^{1 \& 2}$ Student, ${ }^{3 \& 4}$ Assistant Professor,Department of Master of Computer Application, Bharati Vidyapeeth \\ (Deemed to be University)Pune, Institute of Management Kolhapur Maharashtra India
}

\begin{abstract}
:
The World Wide Web, which has started as a document repository, is rapidly transforming to a full fledged virtual environment that facilitates services, interaction, and communication. Under this light, the Semantic Web and Web 2.0 movements can be seen as intermediate steps of a natural evolution towards a new paradigm, the 3D Internet. We provide an overview of the concept 3D Internet and discuss why it is a goal worth pursuing, what it does entail, and how one can realize it. Our goal in this paper is to discuss a research agenda and raise interest in networking, security, distributed computing, and machine learning communities. We explore first the motivation for the 3D Internet and the possibilities it brings. Subsequently, we investigate the specific system level and research challenges that need to be addressed in order to make the 3D Internet a reality.
\end{abstract}

Keywords: 3D Internet, Technology, Networking, Security.

\section{[1] INTRODUCTION}

The Internet is evolving to become the de-facto cyberspace or virtual environment facilitating communication, business, and entertainment on a global scale. On the other hand, met verses or virtual worlds such as Second Life (SL) or World of War craft (WoW) are much younger 
when compared to other Web technologies. Today, the success and momentum of virtual worlds are undeniable. The market for MMOGs is estimated to be worth more than one billion US dollars and such meta verses are fast becoming "significant platforms" in the converged media world according to some analysts. Virtual worlds are increasingly seen as more than game and interpreted within a business context rather than entertainment. The view that Meta verses will play a significant role in the future is shared by many researchers and professionals in the field. Among them are the participants of the Meta verse roadmap (MVR) who aim to explore multiple pathways to the 3D enhanced web [2], the Croquet Consortium [1], as well as the VRML and X3D communities

We envision a 3D Internet which will be to 2D graphical user interface (GUI) and Web of today what 2D GUI and World Wide Web (WWW) were to command line interface (CLI) and gopher two decades ago. While the concept seems incremental in the sense that it merely adds 3D graphics to the current Web, it is in fact revolutionary for it provides a complete virtual environment that facilitates services, interaction, and communication. From this perspective, the 3D Internet can be seen as the evolutionary end point of ongoing efforts such as Web 2.0 and Semantic Web. Our objective in this paper is to define the 3D Internet concept and discuss why it is a goal worth pursuing, what it does entail, and how one can realize it. Along with its enormous potential the 3D Internet also opens many research challenges in order to become a reality. Meta verses have recently caught the attention of gaming, advertisement, 3D design, and performing arts communities among others. However, it is difficult to claim that the same level of interest has been raised in the areas of networking, machine learning, and distributed computing. Without overcoming these engineering challenges and making a business case to stakeholders the 3D Internet is destined to be an academic exercise and remain in the realm of science fiction; a fate experienced by many initially promising concepts such as artificial intelligence or virtual reality. We discuss in the next section why stakeholders such as communication and computing companies, research institutions, and online businesses should be interested and participate in the 3D Internet. In Section 3, we present example architecture as a starting point for the 3D Internet. Section 4 summarizes the engineering challenges and explores research directions in various fields. The paper concludes with remarks in Section 5. 


\section{[2] 3D Internet:}

One of the often heard arguments against the 3D Internet is in the form of the question "why do we need it?" For most of its users the Internet is a familiar, comfortable medium where we communicate with each other, get our news, shop, pay our bills, and more. We are indeed so much used to and dependent on its existence that we don't think about its nature anymore just like we do not think about Ohm's law when we turn on the lights. From this perspective what we have, i.e. the 2D version, seems "sufficient" and the 3D Internet is yet another fad. However, if we stop and think about the nature of the Internet for a moment we realize that it is nothing but a virtual environment (cyberspace) where people and organizations interact with each other and exchange information. Once this fact is well understood, the question can be turned on its head and becomes "why do we restrict ourselves to 2D pages and hyperlinks for all these activities?"

Navigating hierarchical data structures is often cumbersome for large data sets. Unfortunately, the Internet as we know is organized as a flat abstract mesh of interconnected hierarchical documents. A typical 2D website is an extremely abstract entity and consists of nothing but a bunch of documents and pictures. Within the website, at every level of the interaction, the developers have to provide the user immediate navigational help. Otherwise, the user would get lost sooner or later. Since this is a very abstract environment, there is no straightforward way of providing a navigation scheme which would be immediately recognizable to human beings. The situation is not any better when traveling between websites. Although the domain name system is somewhat helpful, using the web today is no different than reading a telephone directory. Given the current situation the term web surfing is rather appropriate as we have no control over where the web takes us with the next click. This has profound implications such as the reliance on back button in browsers which tantamount to admitting that navigating on the web is no different from a random walk. Another consequence is the emergence of search engines as a fundamental element of the Internet. It is no surprise that Google is the most powerful Internet Company of our times.

There is actually a much better alternative way of organizing data which everybody knows and uses. We spend all our lives in a 3D world navigating between places and organizing objects spatially. We rarely need search engines to find what we are looking for and our brains are naturally adept at remembering spatial relationships. Let us consider the following fictitious 
scenario on the 3D Internet. Instead of a flat 2D desktop I can put my documents on my desk at home, where documents, desk, and home are "virtual" entities that are 3D representations of real-world counterparts with spatial relationships. Later, when the need of finding these documents arises, there is a high probability that I can easily remember their location without resorting to additional processes such as search engines or a "recent documents" folder. Obviously, it is very difficult -if not impossible- to realize this scenario on the current Internet. We are there like 2D creatures living on flat documents not knowing where we are or what is next to us. We teleport constantly from one flat surface to another, each time getting lost, each time asking for directions or help. In contrast, the ease of use and intuitiveness of 3D GUIs are an immediate consequence of the way our brains work, a result of a long evolutionary process ensuring adaptation to our world. Although the 3D Internet is not a solution to all problems, it provides an HCI framework that can decrease mental load and open doors to rich, innovative interface designs through spatial relationships. Another important point is the Web place metaphors of the 3D Internet which enables interaction between people in a natural way. In this sense, the 3D Internet can be seen as a natural successor of Web 2.0.

The met verses such as SL can be considered as pioneering precursors of the 3D Internet. Yet, they already indicate its significant business opportunities. Not only existing online businesses would benefit from the inherent interactive nature and spatial HCI paradigms of the 3D Internet but also a whole range of businesses such as fashion, real estate, and tourism can finally start using the Internet effectively. We expect that the possibility of providing faithful 3D representations of products and services will have revolutionary effects on online business to business and business to customer commercial activity. From virtual "try before buy" to “interactive shopping" the commercial potential of the 3D Internet is enormous.

\section{[3] 3D Internet:}

What are the benefits of Internet? We present and discuss a 3D Internet architecture as an illustrative example. It shares the time-tested main principles and underlying architecture of the current Internet as well as many semantic web concepts. The operational principles the 3D Internet shares with its predecessor include open and flexible architecture, open protocols, simplicity at the network core, intelligence at the edges, and distributed implementation. A simple graphical depiction of the proposed 3D Internet architecture is provided in Figure 1. We 
adopt here the terms universe, world, and web place as 3D counterparts of WWW, website, and sub domain, respectively. We describe each components' functionality briefly below: World servers: provide user- or server-side created, static and dynamic content making up the specific web place (3D environment) including visuals, physics engine, avatar data, media, and more to client programs. A world server has the important task of coordinating the co-existence of connected users, initiating communication between them, and ensuring in-world consistency in real time. They may also facilitate various services such as e-mail, instant messaging, and more.

\section{Avatar/ID servers:}

Virtual identity management systems containing identity and avatar information as well as inventory (not only in world graphics but also documents, pictures, e-mails, etc.) of registered users and providing these to individual world servers and relevant client programs (owner, owner's friends) while ensuring privacy and security of stored information. Avatar/ID servers can be part of world servers.

\section{Universe location servers:}

Virtual location management systems similar to and including current DNS providing virtual geographical information as well as connection to the Internet via methods similar to Slur. They can also act as a distributed directory of the world, avatar servers and users.

\section{Clients:}

Extensive networking, caching and 3D rendering capabilities in the browser where the viewer programs running on users computers with Additional components of the 3D Internet include web places (replacing websites) and 3D object creation/editing software, i.e. easy-to-use 3D modelling and design programs such as Sketch-Up and standardized mark-up languages and communication protocols. Emergence of new software and tools in addition to the ones mentioned should naturally be expected.

\section{[4] 3D Internet:}

\subsection{Networking and Distributed Computing}


The conventional web caching approaches will not be adequate for the needs of the $3 \mathrm{D}$ Internet environment consisting of 3D worlds, which may be hosted on different servers. One challenge stems from the fact that avatars contain significantly more information about the user who is visiting a 3D world than cookies do about a 2D web site visitor. For instance, avatars contain information about appearance (e.g. height, clothing) and behaviour (e.g. visible, open for conversation). As avatars move between worlds, caching will be needed in server-to-server interactions to enable fast and responsive transition between worlds. This will be intensified by avatars carrying objects (e.g. a bicycle) or virtual companions (e.g. a virtual dog) with them, which will require the transfer of large volumes of information in a short time when changing world. Another challenge is related to the fact that some virtual objects or companions are essentially not static documents but running programs. They have code that defines how they react to certain inputs, and they have a partly autonomous behaviour. Thus, when an avatar and its companions move to a world, the world server (or servers) needs to execute the corresponding code. This raises a number of interesting research problems: how can we safely run potentially untrusted code (for instance, when the virtual companions are user-generated and custom built)? How will the economics of such transactions be handled? How can we move running code between different world servers without fatally disrupting its execution? Platforms will be needed that allow the dynamic deployment of potentially untrusted computation at globally dispersed servers, in a fast, secure and accountable manner [6].

\subsubsection{Latency Minimization}

As the 3D Internet will increase the reliance on graphics and interactivity, it will be crucial that the latency that clients observe when interacting with servers is minimized. It has been known from existing implementations such as SL that high latency incurs low responsiveness and reduced user satisfaction. Therefore, the network has to be designed intelligently to overcome these challenges. We propose a hybrid peer-to-peer (P2P) approach to reduce server load and ensure scalability of the 3D Internet infrastructure. It consists of three types of communications: client to server $(\mathrm{C} 2 \mathrm{~S})$, server to server $(\mathrm{S} 2 \mathrm{~S})$ and client to client $(\mathrm{C} 2 \mathrm{C})$ each with different latency and bandwidth requirements. C2S communications (see Figure 1 red lines) are bandwidth limited, frequently updated, and synchronous. Location and activity data as well as use of in-world services will spend substantial amount of resources both at the client and world servers. The avatar/ID server-client $\mathrm{C} 2 \mathrm{~S}$ communications (dash-dotted gray lines) are less 
frequent and asynchronous. As an optimization, some portion of this communications can be pushed to the backbone by facilitating S2S links between ID and world servers (solid gray lines) triggered by clients and through intelligent caching. Additional S2S communications will also take place on the backbones. The S2S in the case of universe location servers (dotted gray lines) are expected to be load improving server independent C2C (P2P) communication is one of the main solutions to the scalability problems. One example is the information about avatars in the same space, which can be communicated more efficiently if exchanged directly between the avatars' hosts, instead of through a central server. When the user moves around other avatars can send their information as well as of others within the range in a $\mathrm{P} 2 \mathrm{P}$ fashion as depicted in Figure 2. For example, the avatars in circle L1 can send information about the ones in L2 and them in turn about L3 as a dynamic intelligent caching scheme.

\subsubsection{Security and Trust:}

There is an array of alternatives for enabling the seamless and transparent authentication of users, avatars, and other objects in the 3D Internet world. The Single Sign On concept envisages users logging in only once, for example on a web page of an on-line service, and visiting further services or web-based applications without the need to $\log$ in again. The user can thus experience an unhindered, seamless usage of services. The key concept behind Single Sign On is federation, denoting the establishment of common references between accounts or identities in different repositories or services. Microsoft Passport1 as well as several other systems have been developed based on this concept [9]. Earlier on, role based access control (RBAC) had been devised to allow authentication not based on user identities, but rather based on the class (or classes) they belong to. The studies [5,8] are closer to the 3D Internet paradigm as they focus on challenges imposed by applying RBAC to open, large-scale systems. Attribute-based access control makes access control decisions based on user attributes and their combinations, allowing more fine-grained access control. Driven by the users' growing privacy concerns regarding the handling of their authentication information, user-centric identity management approaches such as CardSpace 2 have recently gained popularity. These go beyond the federation concepts to allow individual users to retain full control over their own identity management, without requiring the presence of an external provider. 


\subsection{Intelligent Environments}

Emerging fields such as ubiquitous computing and ambient intelligence draw heavily from adaptive and intelligent algorithms. They are concerned with computing and networking technology that is unobtrusively embedded in the everyday environment of human users. The emphasis is on user-friendliness, efficient and distributed services support, user empowerment, and support for human interactions. All this assumes a shift away from desktop or portable computers to a variety of devices accessible via intelligent interfaces. The 3D Internet, which is a virtual ubiquitous computing environment, provides the perfect test bed for developing these ideas and emulating them in realistic 3D settings with real users.

\subsubsection{Intelligent Services}

In the case of the 3D Internet, the concept of intelligent environments naturally extends to underlying communication protocols and enabling services as well as to user cantered services. Given its inherent P2P nature, the 3D Internet can make use of paradigms such as intelligent routing where mechanisms being aware of the network topology and information structure allow for flexible and context-dependent distribution of traffic [7]. As in the real world, one could think of adaptive algorithms that control traffic flow depending on the time of day, userbehaviour patterns, or a variety of global and local events.

Since the 3D Internet provides an environment that closely resembles the physical world, it calls for intelligent interfaces that extend the conventional desktop metaphors such as menus and sliders. This may include speech- and gesture recognition, but also implies interaction with virtual objects and tools inspired by things existing in the real world. Learning and ambient intelligence on this level will then have to be concerned with typical usage patterns, anticipations of user activities, and convincing simulations. In terms of user-cantered services, it is not hard to imagine applications of machine learning that would facilitate social interaction of users as well as increase usability of core functionalities of the virtual environments on the 3D Internet. Examples of such services are recommender systems for e-commerce or social networking that rely on collaborative filtering. Based on user provided ratings or an analysis of typical usage patterns, goal directed, intelligent searches [3] and recommendations are possible. This of course facilitates personalization of individual users' avatars and improves multimedia-information retrieval. 


\subsection{2: Intelligent Agents and Rendering}

In order to increase the users' acceptance of services like the ones just mentioned, they will not just have to be personalized but also be presented and accessible in a way users will consider natural. This leads to the problem of modelling artificial agents and avatars [10] that act lifelike and show a behaviour that would be considered natural and human-like. First attempts in this direction have already been made in the context of computer games. Here, machine learning has been shown to provide an auspicious avenue [4]. The network traffic generated by a group of people playing a multiplayer game contains all the data necessary to describe their activities in the virtual game world. Statistical analysis of this traffic and a derivation of a generative model there from allows for implementing agents that are perceived to act more human-like. Corresponding approaches can be applied to improve on the quality of virtual clerks and information personnel.

\section{Conclusions:}

We have provided an overview of the concept 3D Internet and discussed the motivation behind it as well as the specific research directions in the fields of networking, security, distributed computing, and machine learning. We believe that at this point in time we are facing a unique opportunity for the evolution of the Internet towards a much more versatile, interactive, and usable version: the 3D Internet. The emerging 3D applications and desktop paradigms, increasingly interactive nature of the Web 2.0, the Semantic Web efforts, widespread availability of powerful GPUs, popularity of novel input devices, and changing demographics of Internet users towards the younger, computer-literate generations, all provide the basis for the 3D Internet (r)evolution. The hype surrounding Meta verses (especially SL) should be seen under this light and taken as an indicator of the fact that many businesses are aware of the 3D Internet's potential. However, to make the 3D Internet's potential. However, to make the 3D Internet a reality it is necessary and important to start and continue multidisciplinary research.

\section{REFERENCES}

[1] The reference book "The croquet consortium, 2007".

[2] The blog reference "The Meta verse roadmap, 2007".

[3] T. Alpcan, C. Bauckhage, and S. Agarwal. An efficient ontology-based expert peering system. In Proc. 6th IAPR Workshop on Graph-based Repr. in Pattern Recog. (GbR), Alicante, Spain, June 2007. 
[4] B. Gorman, C. Thurau, C. Bauckhage, and M. Humphrys. Believability Testing and Bayesian Imitation in Interactive Computer Games. In Proc. Int. Conf. on Simulation of Adaptive Behavior, September 2006. [5] R. J. Hayton, J. M. Bacon, and K. Moody. Access Control in an Open Distributed Environment. In Proc. IEEE Symp. on Sec. and Priv., May 1998.

[6] E. Kotsovinos, T. Moreton, I. Pratt, R. Ross, K. Fraser, S. Hand, and T. Harris. Global-Scale Service Deployment in the XenoServer Platform. In Proc. Workshop on Real, Large Distributed Systems, December 2004.

[7] M. Li, W.-C. Lee, and A. Sivasubramaniam. Semantic Small World: An Overlay Network for Peer to-Peer Search . In Proc. Int. Conf. on Networking Protocols (ICNP), December 2004.

[8] N. Li, J. C. Mitchell, and W. H. Winsborough. Design of a Role-Based Trust Management Framework. In Proc. IEEE Symp. on Sec. and Priv., May 2002.

[9] A. Pashalidis and C. Mitchell. A taxonomy of single sign-on systems. In Proc. 8th Australasian Conf. in Inf. Sec. and Pri. (ACISP), July 2003.

[10] M. Wooldridge and N. R. Jennings. Intelligent Agents: Theory and Practice. The Knowledge Engineering Review, 10(2):115-152, 1995.

\section{Author[s] brief Introduction}

1.Tejas Sawant- Student of MCA-II (Sem- III), Bharati Vidyapeeth (Deemed to be University) Institute of Management Kolhapur. Mail:Tejassawant1149@yahoo.com

2.Sarang Gulavani- Student of MCA-II (Sem- III), Bharati Vidyapeeth (Deemed to be University) Institute of Management Kolhapur. Mail:saranggulavani.mj@gmail.com

3.Dr.Bhaskar Patil- Assistant Professor Bharati Vidyapeeth (Deemed to be University) Institute of Management Kolhapur. Mail:bhaskarpatil@bharatividyapeeth.edu

4.Prof.Nripesh.Nirp- Assistant Professor Bharati Vidyapeeth (Deemed to be University) Institute of Management Kolhapur. Mail:nirpesh.nrip@gmail.com 